\title{
Observation of Density Fluctuations Originated from RF Waves with Two-Channel Reflectometer in GAMMA 10/PDX ${ }^{*)}$
}

\author{
Ryo SEKINE, Ryuya IKEZOE ${ }^{1)}$, Seowon JANG, Mafumi HIRATA, Makoto ICHIMURA, \\ Mizuki SAKAMOTO, Shuhei SUMIDA, Koki IZUMI, Atsuto TANAKA, Yushi KUBOTA, \\ Hiroki KAYANO and Yousuke NAKASHIMA \\ Plasma Research Center, University of Tsukuba, Tsukuba 305-8577, Japan \\ ${ }^{1)}$ Research Institute for Applied Mechanics, Kyushu University, Kasuga 816-8580, Japan
}

(Received 30 September 2018 / Accepted 30 November 2018)

\begin{abstract}
Slow Alfvén wave with a frequency just below an ion cyclotron frequency can be destabilized by the anisotropy of ion temperature and called as Alfvén-Ion-Cyclotron (AIC) wave. In the GAMMA 10/PDX central cell, several AIC waves simultaneously appear in an unstable frequency range. In addition, low-frequency waves with the difference frequencies (DF) between the AIC waves are observed, and their characteristics have to be clarified to reveal their unknown contributions to the ion transport suggested in GAMMA 10/PDX. In this study, simultaneous two-point measurements in the azimuthal and axial directions were conducted by using a two-channel microwave reflectometer with multi horn antennas. The azimuthal mode number $(m)$ of the DF waves was confirmed at inner plasma region for the first time, and was zero, which satisfies azimuthal mode matching with the $m=-1$ AIC waves. In addition, it was shown that the DF waves were radially localized in an inner region, which is different from rather broad profiles of the AIC waves.
\end{abstract}

(C) 2019 The Japan Society of Plasma Science and Nuclear Fusion Research

Keywords: GAMMA 10/PDX, ICRF heating, AIC wave, microwave reflectometer, low-frequency wave

DOI: $10.1585 /$ pfr. 14.2402011

\section{Introduction}

There are many types of nonlinear phenomena in high-temperature plasmas for nuclear fusion experiments. In the case of a plasma heating with a high frequency wave, nonlinear phenomenon plays an important role in heating efficiency. Parametric instability, for example, is a wellknown concern for a high-power wave heating [1].

In the GAMMA 10/PDX, ion cyclotron range of frequencies (ICRF) waves have been used for plasma production and heating [2]. The maximum ion temperature reached is about $10 \mathrm{keV}$, and the temperature anisotropy (which is defined as the temperature ratio of the component perpendicular to the magnetic field line to the parallel component) is greater than 10 . Due to such a strong temperature anisotropy, Alfvén-Ion-Cyclotron (AIC) wave is excited at a frequency just below the ion cyclotron frequency [3]. In the GAMMA 10/PDX central cell, several AIC waves simultaneously appear, and the differencefrequencies (DF) between these frequencies are about $100 \mathrm{kHz}$. It is clearly observed by a microwave reflectometer that the DF waves are nonlinearly excited via the coupling between the AIC waves especially in a core region of the central cell [4]. In addition, it has been observed that high-energy ions are periodically transported to the end region along the magnetic field lines with the frequencies of

author'se-mail: sekine_ryo@prc.tsukuba.ac.jp

*) This article is based on the presentation at the 12th International Conference on Open Magnetic Systems for Plasma Confinement (OS2018). the DF waves. Therefore, the DF waves are believed to contribute to axial transport of high-energy ions [5]. However, the spatial structure and the physical characteristics of the DF waves are unknown.

Simultaneous two-point measurements of the density fluctuations accompanied by the waves in both the azimuthal and axial directions were conducted by using a two-channel microwave reflectometer with multi horn antennas. The obtained phase profiles of the DF and AIC waves are shown along with their radial intensity profiles.

\section{Two-Channel Microwave Reflec- tometer}

A reflectometer, which we use for the wave detection, is a useful tool for investigation of density fluctuations inside a hot plasma in spite of a relatively small-sized measurement system. The two-channel microwave reflectometer system used in this study is composed of two simple heterodyne reflectometers and utilizes two independent frequency-variable yttrium iron garnet (YIG) oscillators to create two probing microwaves [6].

Six horn-antenna pairs are installed in the interior of the vacuum vessel of the central cell; five of them are arranged along a magnetic field line $(z=0.52,0.82,1.12$, 1.42 and $1.93 \mathrm{~m}$ ) [6], and the rest pair has been newly installed for this study at $z=0.82 \mathrm{~m}$ with the azimuthal interval of 78 degrees with the axial array. The antenna po- 
(a)

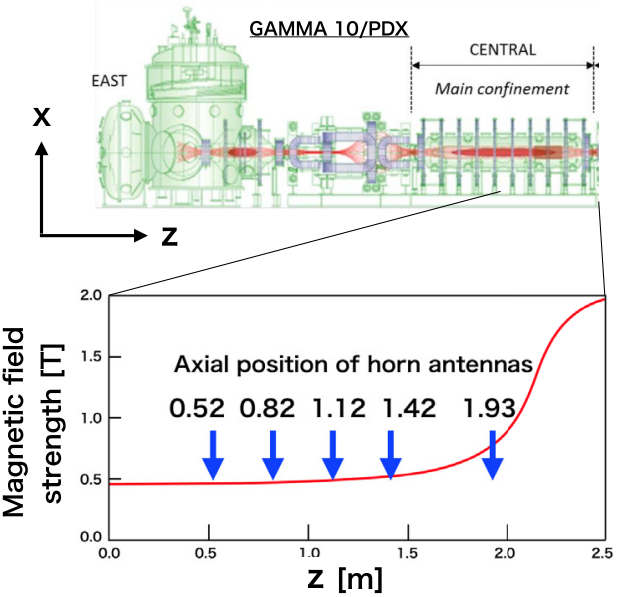

(b)

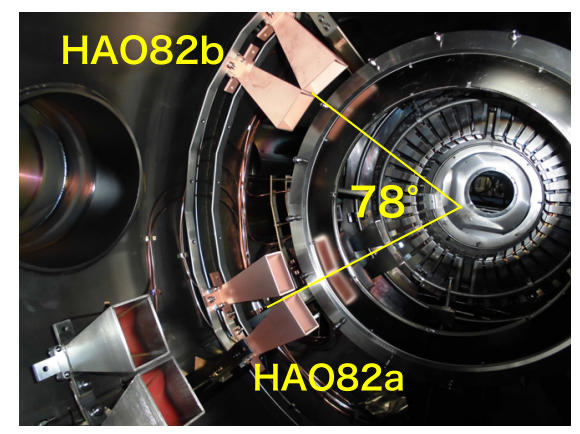

Fig. 1 (a) Schematics of GAMMA 10/PDX and the axial locations of the horn-antennas with the axial profile of the field strength. (b) A photograph showing the interior of the vacuum vessel.

sitions are schematically shown in Fig. 1. This system can be used to measure density fluctuations either at axially separated two positions or azimuthally separated two positions at the same time. In addition, measurement in a radial direction can be achieved by changing the incident frequency. The frequency range of applicable microwaves is about 8.0 to $13 \mathrm{GHz}$ (X-band). The typical electron density in GAMMA 10/PDX has a maximum of about $2 \times 10^{18} \mathrm{~m}^{-3}$ at the center and monotonically decreases toward the edge, and $\mathrm{X}$-band $\mathrm{O}$-mode microwaves injected from the outside into the plasma cover a relatively wide radial region for reflectometry.

\section{Experimental Results and Discus- sion}

\subsection{Frequency spectrum analysis}

The plasma in the central cell is mainly heated by exciting ICRF wave (by RF2 system) with a fundamental ion cyclotron resonance frequency near the midplane of the central cell. Figure 2 shows the temporal evolution of (a) the RF power (RF2) and (b) the line density at $z=-0.60 \mathrm{~m}$ and the diamagnetic signal at $z=-0.33 \mathrm{~m}$. Figure 3 shows the frequency spectrum of the density fluctuations obtained with the incident microwave frequencies (a)

(b)

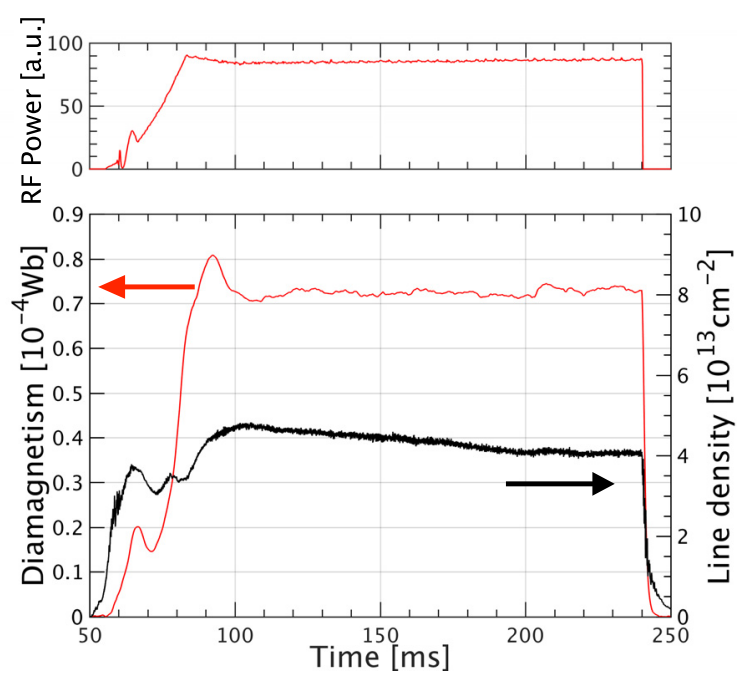

Fig. 2 The temporal evolution of (a) the ICRF pulse (RF2), and (b) the line density $(z=-0.60 \mathrm{~m})$ and the diamagnetism $(z=-0.33 \mathrm{~m})$ in the central cell.

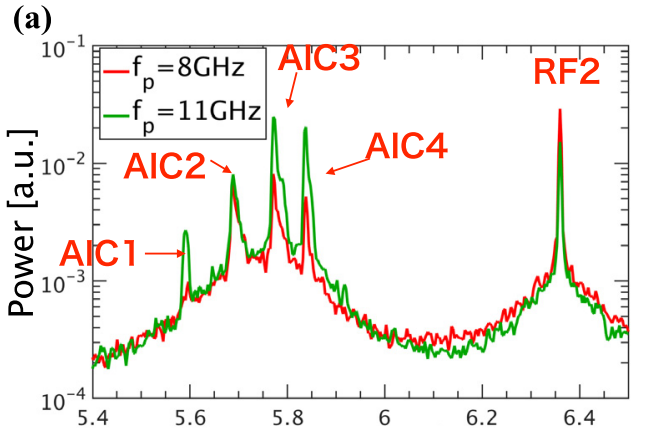

(b)

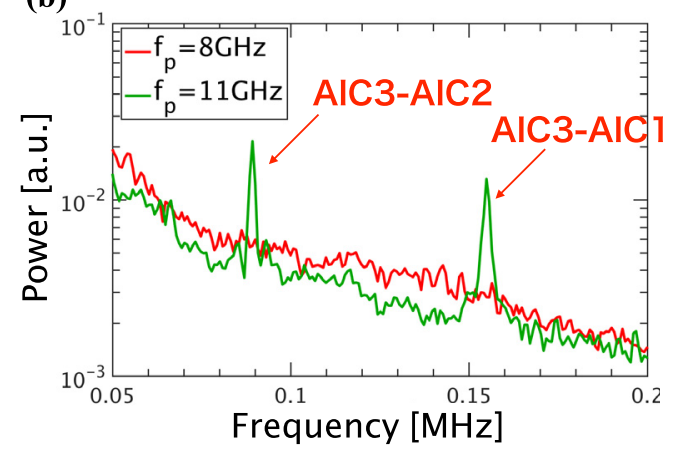

Fig. 3 Frequency spectra of the density fluctuations measured with the reflectometer in frequency ranges (a) 5.4 $6.5 \mathrm{MHz}$ and (b) $0.05-0.2 \mathrm{MHz}$. The microwave frequency is $8.0 \mathrm{GHz}$ for red line and $11.0 \mathrm{GHz}$ for green line, and the horn antenna pair at $z=82 \mathrm{~cm}$ is used.

of $8 \mathrm{GHz}$ (peripheral side of plasma) and $11 \mathrm{GHz}$ (core side of plasma) at $z=0.82 \mathrm{~m}$; where the spectra are time averaged between 190 and $200 \mathrm{~ms}$. As the RF power for heating is increased, the diamagnetism increases and the temperature anisotropy becomes strong, and the AIC waves are spontaneously excited from about $80 \mathrm{~ms}$. In 


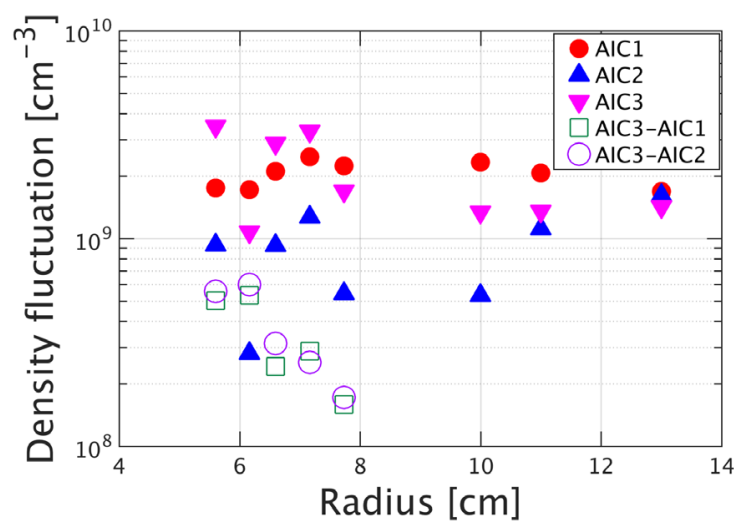

Fig. 4 Radial distribution of density fluctuation intensity obtained for the AIC waves and the difference-frequency waves.

Fig. 3 (a), several frequency peaks corresponding to the AIC waves $(5.6-5.9 \mathrm{MHz})$ are observed in both radial positions. A peak at $6.36 \mathrm{MHz}$ corresponds to the applied ICRF wave (RF2). Figure 3 (b) shows the frequency spectrum in the frequency band from 50 to $200 \mathrm{kHz}$, in which the difference-frequency (DF) waves of the AIC waves are clearly observed. Two peaks (around 90 and $150 \mathrm{kHz}$ ) are observed in the case of $f_{p}=11 \mathrm{GHz}$ while no peak is observed in the case of $f_{p}=8 \mathrm{GHz}$. From these results, it is considered that the DF waves are not excited in a whole plasma radius.

\subsection{Radial distribution of density fluctuation intensity}

Radial scanning of the intensity of these density fluctuations has been carried out by varying the incident microwave frequency from 8.0 to $11.6 \mathrm{GHz}$ shot-to-shot in a series of identical discharges; eight cutoff positions were probed in total. Figure 4 shows radial distributions of the intensity of density fluctuations accompanied by each AIC wave and each DF wave, where each AIC wave and DF wave is numbered in the same manner as in Fig. 3. The density fluctuation intensities of the AIC waves and the DF waves are calculated by considering the density gradient;

$$
\tilde{n_{c}}=\frac{\mathrm{d} n_{e}}{\mathrm{~d} r} \lambda \frac{\Delta \phi}{4 \pi},
$$

where $\tilde{n_{c}}$ shows the density fluctuation. $\mathrm{d} n_{e} / \mathrm{d} r$ and $\Delta \phi$ are the electron density gradient and the phase fluctuation, respectively. $\lambda$ is the effective wavelength of a microwave; here, we used 1.7 times longer than the vacuum wavelength [7]. The density gradient and the radial position are calculated from the density profile that is obtained by using a radially scanning microwave interferometer system. The phase fluctuation intensity in the vertical axis in Fig. 4 is subtracted by the background noise since rather intense background fluctuations which have a radial dependence are superimposed especially in a low-frequency
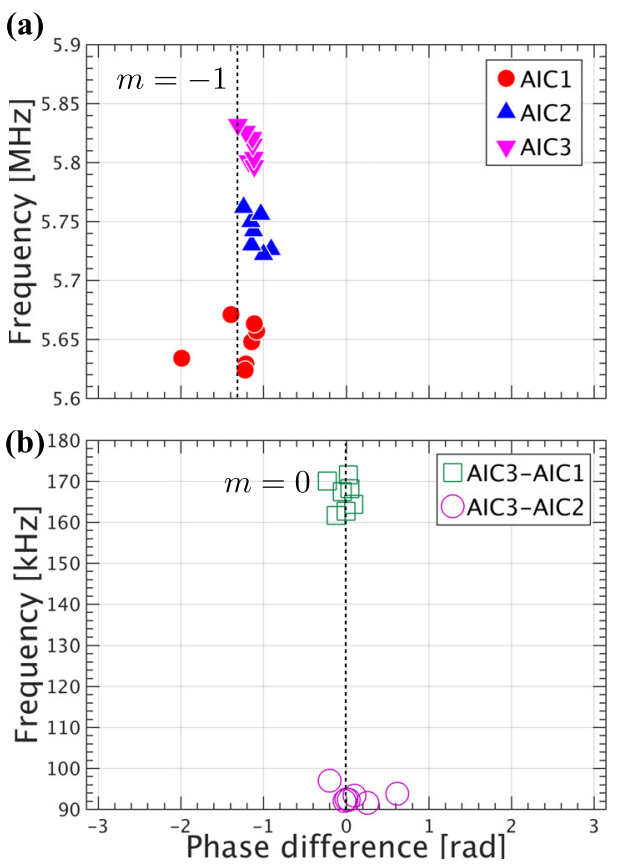

Fig. 5 Azimuthal mode structures of (a) the AIC waves and (b) the difference-frequency waves, in the case of $f_{p}=$ $11 \mathrm{GHz}$.

band. Then, it is possible to compare the data between different radial positions as well as the data on the same radial position.

As shown in Fig. 4, the DF waves have a different radial profile with lower amplitudes compared with the AIC waves. The AIC waves have a somewhat broad profile with a significant amplitude of about $10^{-3}$ of the electron density while the DF waves are only significant at $r<8 \mathrm{~cm}$, and drops to the noise level in the outer side. In addition, it is seen that the fluctuation intensities of the DF waves increase toward the inner side. Therefore, it is considered that the DF waves are localized in a core region, supporting the result shown in Ref. [4], which indicates strong nonlinear coupling among the AIC waves in a core region.

\subsection{Phase difference measurement}

Spatial structures of the AIC and DF waves are investigated by using the two-channel reflectometer with hornantenna pairs arrayed in the azimuthal and axial directions. Figure 5 shows the azimuthal phase difference of each peak of the AIC waves and the DF waves measured with the antenna pairs having the azimuthal angle of 78 degrees at $z=0.82 \mathrm{~m}$. The incident microwave frequency is $11 \mathrm{GHz}$, so the region where the DF waves are excited with a significant amplitude is measured. The azimuthal mode numbers are estimated from the phase differences between the antenna pairs by

$$
m=\frac{\Delta \phi}{\Delta \phi_{H A}},
$$

where $\Delta \phi$ and $\Delta \phi_{H A}$ are the measured azimuthal phase dif- 

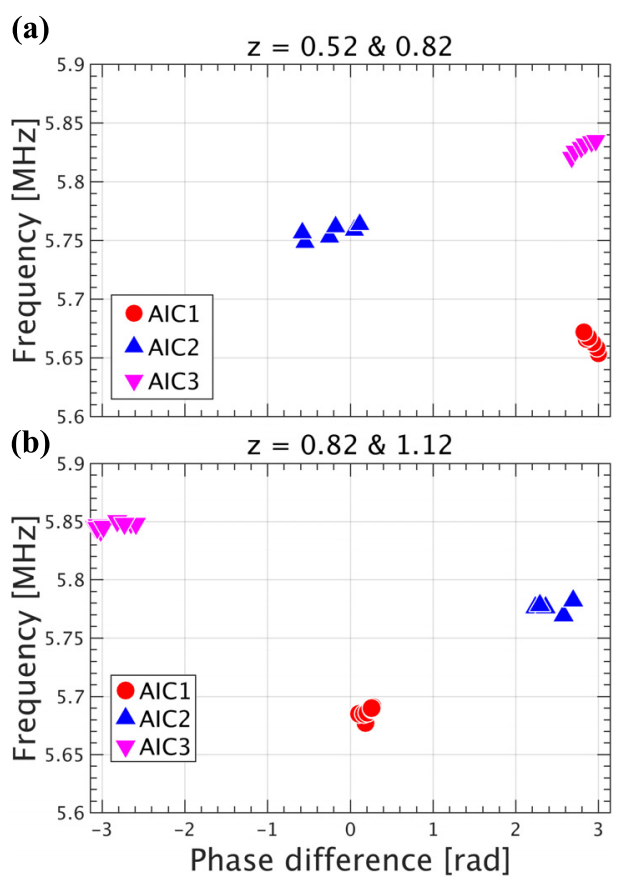

Fig. 6 Axial phase difference of the AIC waves at (a) $z=$ $0.52 \& 0.82 \mathrm{~m}$, and (b) $z=0.82 \& 1.12 \mathrm{~m}$.

ference and the azimuthal angle difference between hornantenna pairs $\left(\Delta \phi_{H A}=78 \mathrm{deg} .=1.36 \mathrm{rad}.\right)$, respectively. $m$ is azimuthal mode number, and positive $m$ indicates that a wave propagates in the direction of electrondiamagnetic drift motion. In Fig. 5, the phase difference has been plotted for the peak frequency of the waves, and for the time period when the wave had a high coherence between the two points. The phase differences between the waves are almost constant during the measurements as shown in Fig. 5. The AIC waves are detected as $m=-1$, which coincides with the previous result measured by a magnetic probe pair at the plasma edge; the AIC waves are surely slow Alfvén waves [8]. On the other hand, the DF waves are detected as $m=0$. Thus, the azimuthal mode matching which is necessary for three-wave coupling is satisfied; the difference interaction of two AIC waves of $m=-1$ produces $m=0$. When the frequency of the incident microwave was changed, the same azimuthal phase difference as in the case of $f_{p}=11 \mathrm{GHz}$ have been confirmed.

A phase difference measurement with two-channel reflectometer in the axial direction has been also carried out under the similar discharge conditions. Figure 6 shows the axial phase difference of each peak of the AIC waves at $z=0.52 \& 0.82 \mathrm{~m}$, and $z=0.82 \& 1.12 \mathrm{~m}$. In Fig. 6 , the axial phase difference of the AIC waves shows 0 or $\pm \pi$ rad. In the previous paper [9], it is reported that the AIC waves form standing waves near the midplane of the central cell, and propagate in the outside of the standing wave region. It is shown from Fig. 6 that in the time period analyzed here, AIC1 has a standing wave node between $z=$ 0.52 and $0.82 \mathrm{~m}$ while the node of AIC2 exists in between $z=0.82$ and $1.12 \mathrm{~m}$. In this manner, the two-channel microwave reflectometer clearly measured the axial structure of the AIC waves while, unfortunately, the axial structure of the DF waves was not evaluated due to those low coherence.

\section{Summary}

In the GAMMA 10/PDX central cell, density fluctuations originated from RF waves have been measured by using a two-channel microwave reflectometer. Spontaneous excitation of the AIC waves and the low frequency waves with the difference-frequencies between the AIC waves are observed clearly. Measurement of the radial intensity profile of each wave has been performed by changing the incident microwave frequency. It is clarified that the DF waves are localized in a core region while the AIC waves are excited broadly in the radial direction. Furthermore, by measuring the phase differences of these waves between the two points separated in the azimuthal direction, it is found that the azimuthal mode number of the DF waves is $m=0$, which satisfies the azimuthal mode matching necessary for the three-wave interaction with two $m=-1$ AIC waves.

\section{Acknowledgments}

The authors acknowledge the member of Plasma Research Center in University of Tsukuba for their collaboration. This work was partly supported by the bidirectional collaborative research program of the National Institute for Fusion Science, Japan (NIFS14KUGM086 and NIFS17KUGM132).

[1] M. Porkolab and R. Chang, Rev. Mod. Phys. 50, 745 (1978).

[2] M. Ichimura et al, Plasma Phys. Rep. 28, 727 (2002).

[3] M. Ichimura et al, Phys. Rev. Lett. 70, 2734 (1993).

[4] R. Ikezoe et al, Phys. Plasmas 22, 090701 (2015).

[5] R. Ikezoe et al, Nucl. Fusion 53, 073040 (2013).

[6] R. Ikezoe et al, Rev. Sci. Instrum. 88, 033504 (2017).

[7] A.E. Costley, "Diagnosis of Fusion Plasmas Using Reflectometry", in International School of Plasma Phys. p.379-396 (1986).

[8] M. Ichimura et al, J. Plasma Fusion Res. SERIES 8, 893 (2009).

[9] R. Katsumata et al, Phys. Plasmas 3, 4485 (1996). 